\title{
FORO
}

\section{Sobre la presencia de Paraptenodytes y Palaeospheniscus (Aves: Sphenisciformes) en la Formación Bahía Inglesa, Chile}

\author{
On the precense of Paraptenodytes y Palaeospheniscus \\ (Aves: Sphenisciformes) on the Bahia Inglesa Formation, Chile
}

MARTÍN F. CHÁVEZ

Instituto de Zoología, Universidad Austral de Chile, Campus Isla Teja, Valdivia, Chile e-mail:paleoaeolos@gmail.com

\begin{abstract}
RESUMEN
El presente comentario fue motivado por el artículo de Acosta-Hospitaleche \& Canto (2005) y de la observación directa de algunos especímenes de Spheniscidae previamente reportados para la Formación Bahía Inglesa, en la región de Atacama, Chile. La falta de caracteres morfológicos que permitan la diferenciación con el género Spheniscus y de restos diagnósticos asociados, descartan la asignación de materiales craneales a Palaeospheniscus. Igualmente no es posible corroborar la asignación de especímenes a Paraptenodytes, sugiriéndose el uso de Spheniscidae indet. aff. Paraptenodytes para un tarsometatarso aislado. Se sugiere también el uso de Spheniscus spp. para los especímenes previamente referidos a $S$. cf. chilensis y $S$. aff. humboldti. De este modo, el número de pingüinos registrados en la formación se reduce de nueve a siete.
\end{abstract}

Palabras clave: Palaeospheniscus, Paraptenodytes, Spheniscus, Formación Bahía Inglesa, pingüinos fósiles.

\begin{abstract}
The present comment was motivated by the article by Acosta-Hospitaleche \& Canto (2005), and from the direct observation of some specimens of Sphenicidae previously reported for the Bahia Inglesa Formation, in the Atacama region, Chile. The lack of morphological characters that they allow the differentiation with the genus Spheniscus and of associate diagnostic remains discard the assignment of cranial materials to Palaeospheniscus. Equally it is not possible to corroborate the assignment of specimens to Paraptenodytes, being suggested the use of Spheniscidae indet. aff. Paraptenodytes for an isolated tarsometatarsus. It is also suggested the use of Spheniscus spp. for the specimens previously referred to $S$. cf. chilensis and $S$. aff. humboldti. This way, the number of penguins registered in the formation decreases from nine to seven.
\end{abstract}

Key words: Palaeospheniscus, Paraptenodytes, Spheniscus, Bahia Inglesa Formation, fossil penguins.

El inicio de la presente década coincidió con un aumento substancial en nuestro conocimiento del registro fósil chileno, particularmente en el caso de la avifauna marina del Neógeno. En este sentido, el aporte de la Formación Bahía Inglesa (Mioceno medio-Plioceno tardío) en la región de Atacama, es incuestionable, ofreciendo un amplio registro que incluye seis familias de aves marinas, siendo los Spheniscidae los mejor representados (Chávez $2005)^{1}$. El presente comentario nace tanto de la

1 CHÁVEZ M (2005) Las aves fósiles de Chile y Península Antártica. Resúmenes del Segundo Congreso Latinoamericano de Paleontología de Vertebrados, Río de Janeiro, Brasil. Boletim de Resumos: 79-80. observación directa de algunos especímenes de Spheniscidae previamente reportados, como de la reciente publicación de Acosta-Hospitaleche \& Canto (2005), la cual presenta incongruencias que requieren de una pronta corrección. En el presente se mencionan especímenes pertenecientes a las colecciones de Paleontología de Vertebrados del Museo Nacional de Historia Natural (SGO-PV), Santiago, Chile; del Museo Paleontológico de Caldera (MPC), Atacama, Chile; y del Museo de la Universidad Nacional Mayor de San Marcos (MUSM), Lima, Perú.

Hasta la fecha se conoce un total de nueve taxones reportados para la Formación Bahía 
Inglesa (Chávez $2005^{1} \mathrm{Walsh} 2004^{2}$ ). El primer estudio sobre la avifauna de la formación fue el realizado por Fritis (2001), quien distingue seis taxones y postula por primera vez la existencia de los géneros Palaeospheniscus y Paraptenodytes, descritos originalmente para el Mioceno temprano de Argentina; junto con un nuevo taxón afín a Pygoscelis. Fritis fue el primero en asociar materiales craneales al género Palaeospheniscus, sin embargo, reconoce una estrecha similitud morfológica con el género Spheniscus, derivándolos por considerar que Spheniscus solo aparecería a partir del Plioceno tardío. Los resultados de esta primera aproximación son cuestionables, dado que no se realizan descripciones detalladas de los materiales ni una verificación de la diagnosis para los taxones reportados.

Posteriormente, Acosta-Hospitaleche et al. $(2002)^{3}$ suplen las falencias del trabajo anterior y presentan un total de cuatro taxones, conservando el uso de Paraptenodytes antarcticus para elementos apendiculares y de Palaeospheniscus sp. para los elementos craneales, confirmando la presencia del género Pygoscelis y reportando por primera vez la presencia de Paraptenodytes robustus. Sin embargo, por tratarse de un resumen, no se amplían las descripciones de los materiales y por tanto no aporta a la corroboración de las identificaciones. Las especies ofrecidas en este trabajo, han sido acogidas en la mayoría de las revisiones posteriores (Acosta-Hospitaleche \& Tambussi $2004^{4}$, Walsh $2004^{2}$, Chávez $2005^{1}$, Tambussi et al. $2005^{5}$ ).

WALSH S (2004) New penguin remains from the Neogene of Chile. Sixth International Meeting of the Society of Avian Paleontology and Evolution, Quillan, France. Book of Abstracts: 60-61.

3 ACOSTA-HOSPITALECHE C, O FRITIS, C TAMBUSSI \& A QUINZIO (2002) Nuevos restos de pingüinos (Aves Spheniscidae) en la Formación Bahía Inglesa (Mioceno superior- Plioceno inferior) de Chile. Actas del Primer Congreso Latinoamericano de Paleontología de Vertebrados, Santiago, Chile. Libro de Resúmenes: 14.

4 ACOSTA-HOSPITALECHE C \& C TAMBUSSI (2004) Fossil penguins from South America. Fifth International Conference on Penguin, Ushuaia, Argentina. Book of Abstracts: 48.

5 TAMBUSSI C, C ACOSTA-HOSPITALECHE \& J CANTO (2005) Paleornitofauna de pingüinos de Chile. Resúmenes del Segundo Congreso Latinoamericano de Paleontología de Vertebrados, Río de Janeiro, Brasil. Boletim de Resumos: 259.
De la observación directa es posible constatar que el estado de conservación de algunos especímenes estudiados por Fritis (2001) y Acosta-Hospitaleche et al. (2005) impiden la verificación de las diagnosis conocidas para el género Paraptenodytes (sensu Acosta-Hospitaleche 2004). Se revisaron los dos fémures asignados a $P$. antarcticus. En el espécimen MPC 1022 (previamente citado como MC 108) la fosa poplitea se encuentra cubierta por sedimentos y la cresta trocleante está mal conservada. Si bien presenta una depresión supra sulco platelar marcada como en Paraptenodytes, la diáfisis en general presenta una superficie irregular que sugiere que estas depresiones pueden tener un origen no morfológico (i.e. erosión, interacción con predadores). La cresta tibiofibular de borde filoso, la fosa trocante profunda y la cabeza ligeramente más baja que el trocante difiere de lo descrito en Paraptenodytes. En MPC 1023 (previamente citado como MC 109) la fosa poplitea es abierta y no dividida como en Paraptenodytes, tampoco se observa la depresión supra sulco platelar y la cabeza está poco diferenciada de la diáfisis. Con base en lo anterior, no es posible confirmar la asignación a $P$. antarcticus.

De los especímenes revisados solo el tarsometatarso MPC 1024 (previamente citado como MC 104), presenta algunos rasgos afines con el morfotipo paraptenodytino: índice de elongación menor a $2(1.8)$ y tróclea del digito II divergente en dirección medial; mientras que en el tarsometatarso MPC 1025 (previamente citado como MC 105) no se observan caracteres distintivos de dicho morfotipo, presentando mayor similitud con el género Spheniscus. De momento se sugiere la designación aff. Paraptenodytes solo para el tarsometatarso MPC 1024 a la espera de una revisión crítica.

Más recientemente, Acosta-Hospitaleche \& Canto (2005) confieren nuevos cráneos aislados al género Palaeospheniscus. La identificación genérica de los especímenes hallados en Bahía Inglesa, se justificaría por: (i) ser morfológicamente distinguibles de los géneros Paraptenodytes, Spheniscus y Pygoscelis; (ii) la abundancia de elementos apendiculares del género Palaeospheniscus; y (iii) su coincidencia en el rango de tamaño esperado para los elementos apendiculares del género Palaeospheniscus. Con base en lo anterior, los 
autores concluyen que: "aunque posible, resulta sumamente improbable que estos pertenezcan a una especie diferente o aún no conocida" (494).

Considerando estos fundamentos, se estima que la propuesta taxonómica es injustificada, dado que: (i) todos los caracteres morfológicos mencionados por Acosta-Hospitaleche \& Canto (2005) son compartidos con especies de Spheniscus (Fig. 1). La configuración de las crestas nuchalis temporalis y sagitalis, la fosa de la glándula de sal abierta lateralmente, la morfología de la región temporal y de los procesos paraoccipitales, junto con la forma triangular del techo craneal en vista dorsal; son similares a lo observado en las especies modernas de Spheniscus. Así también, la existencia de frontales fuertemente comprimidos a nivel de las órbitas, una cresta nuchalis sagitalis extensa y de una región occipital en forma trapezoidal; han sido descritas para las paleoespecies Spheniscus urbinai y S. megaramphus (Stucchi 2002, Stucchi et al. 2003). De modo que con base en la descripción ofrecida por los autores, los especímenes son morfológicamente indistinguibles del género Spheniscus. (ii) Los reportes previos del género Palaeospheniscus en la Formación Bahía Inglesa, se han basado únicamente en cráneos, dado que los elementos postcraneales asignados inicialmente (Fritis 2001) fueron reasignados al género Paraptenodytes $^{3}$. Por lo tanto, no existen descripciones de elementos apendiculares diagnósticos para el género en la formación hasta la fecha. (iii) La mayor parte de los materiales postcraneales presentados para la formación, han sido referidos a Spheniscus (Walsh \& Hume 2001)2 (Fig. 2). Los cráneos descritos por Acosta-Hospitaleche \& Canto (2005) coinciden en el rango de tamaño estimado para los elementos apendiculares más abundantes en la formación, los que se atribuyen al género Spheniscus y en ningún caso al género Palaeospheniscus (Fig. 2).

Adicionalmente, Acosta-Hospitaleche \& Canto (2005) señalan que el tamaño de los cráneos es "apenas mayor al de $S$. magallanicus y mucho menor al de Spheniscus urbinai y $S$. megaramphus" (492-493), sin embargo, no contrastan las escasas medidas que ofrecen. Como se aprecia en la presente tabla, la situación es diferente a la descrita por los autores, puesto que los especímenes se encuentran en el rango de tamaño de las especies peruanas y son considerablemente más grandes que S. magallanicus. Al evaluar las medidas ofrecidas para el único espécimen ilustrado en la publicación (SGO-PV 1063), se obtuvieron
(A)

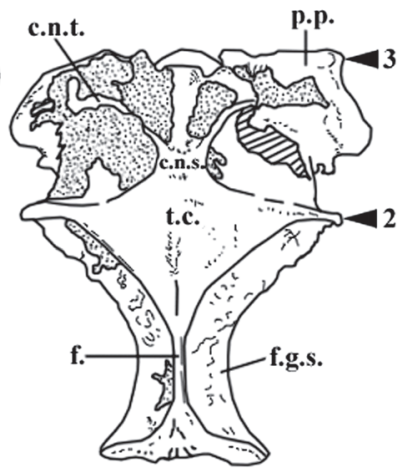

(B)

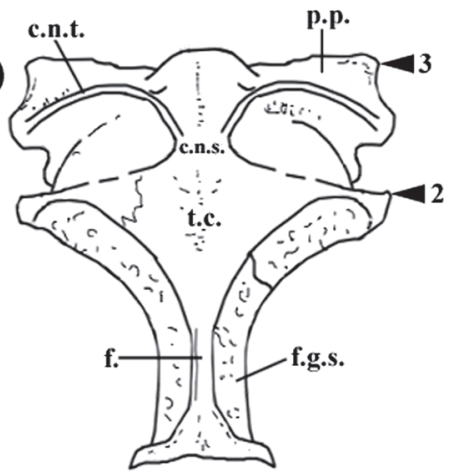

(C)

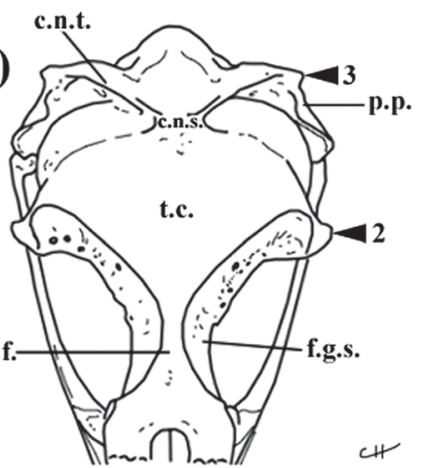

Fig. 1: Comparación de cráneos de Spheniscidae: (A) cf. Palaeospheniscus (SGO-PV 1063), (B) Spheniscus megaramphus (basado en MUSM 175) y (C) S. demersus. Se indica mediante flechas el nivel aproximado de las medidas 2 y 3 ofrecidas en la tabla. Nótese la similitud entre (A) y (B). Abreviaturas: $\mathrm{cns}=$ cresta nuchalis sagitalis, $\mathrm{cns}=$ cresta nuchalis temporales, $\mathrm{f}=$ frontales, $\mathrm{fgs}=$ fosa de la glándula de sal, $\mathrm{pp}=$ proceso paraoccipital, $\mathrm{tc}=$ techo craneal. Ilustraciones de Martín Chávez.

Comparison of spheniscids skulls: (A) cf. Palaeospheniscus (SGO-PV 1063), (B) Spheniscus megaramphus (based in MUSM 175) and (C) S. demersus. The approximate level of the measures 2 and 3 offered in the table its indicated by arrows. See the similarity among (A) and (B). Abbreviations: c.n.s., crista nuchalis sagitalis; c.n.s., crista nuchalis temporalis; f., frontals; f.g.s., supraorbital gland fossae; p.p., paraoccipital process; and t.c., crown of the skull. Ilustrations by Martín Chávez. 


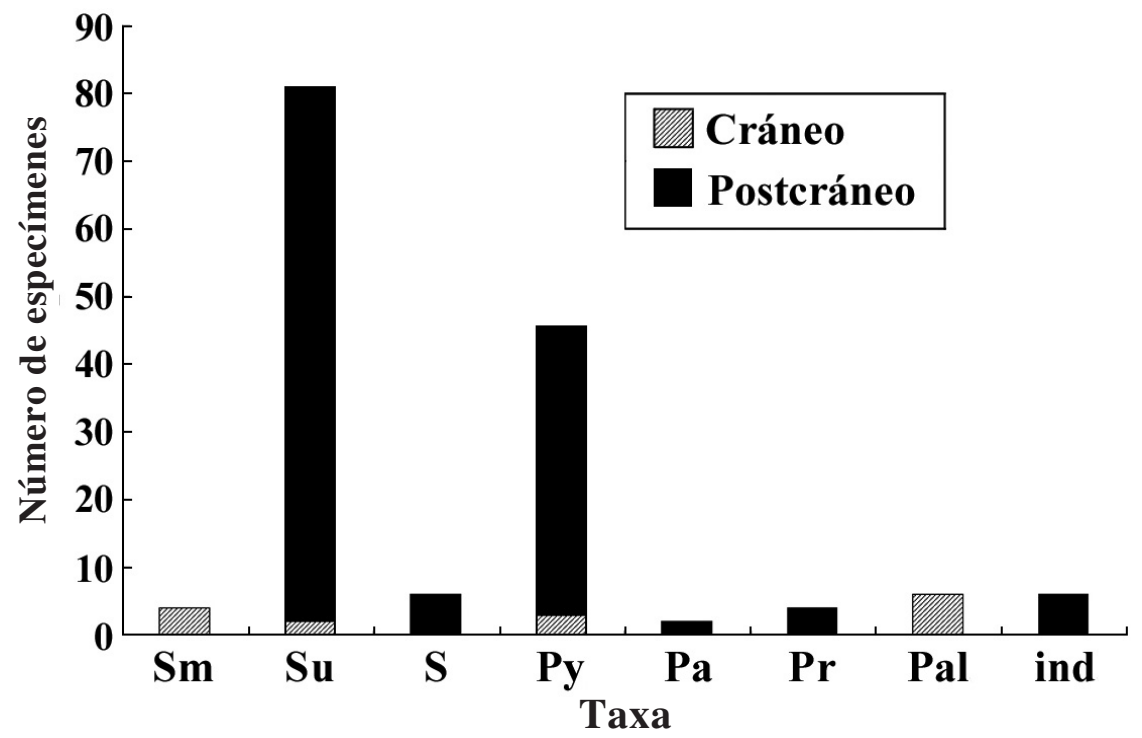

Fig. 2: Número de especímenes de Spheniscidae reportados para la Formación Bahía Inglesa. Se separan los elementos craneales de los postcraneales. Fuente de los datos: Spheniscus y Spheniscidae indeterminado según Walsh \& Hume (2001)2, 6; Pygoscelis según Acosta-Hospitaleche et al. (2006) y Walsh \& Suárez (2006); Paraptenodytes según Acosta-Hospitaleche et al. (2002)3; cf. Palaeospheniscus según Acosta-Hospitaleche \& Canto (2005). Se incorporan además los elementos aquí descritos. Abreviaturas: $\mathrm{Sm}=$ Spheniscus megaramphus, $\mathrm{Su}=S$. urbinai, $\mathrm{S}=S$. spp., $\mathrm{Py}=$ Pygoscelis spp., $\mathrm{Pa}=$ Paraptenodytes antarcticus, $\mathrm{Pr}=$ P. robustus, $\mathrm{Pal}=\mathrm{cf}$. Palaeospheniscus, ind $=$ Spheniscidae indeterminado.

Number of specimens of Spheniscidae reported for the Bahía Inglesa formation. They separate the cranial elements of the postcraneals. Source of the data: Spheniscus and Spheniscidae indeterminate sensu Walsh \& Hume $(2001)^{2}, 6$; Pygoscelis sensu Acosta-Hospitaleche et al. (2006) and Walsh \& Suarez (2006); Paraptenodytes sensu Acosta-Hospitaleche et al. ${ }^{3}$; cf. Palaeospheniscus sensu Acosta-Hospitaleche \& Canto (2005). They also incorporate the elements here described. Abbreviations: S.m., Spheniscus megaramphus; Su, S. urbinai; S, S. spp.; Py, Pygoscelis spp.; Pa, Paraptenodytes antarcticus; Pr, P. robustus; Pal, cf. Palaeospheniscus; and ind, Spheniscidae indeterminate.

TABLA 1

Comparación de medidas e índices craneales de Spheniscus y de un espécimen conferido a Palaeospheniscus. Medidas para el género Spheniscus según Stucchi (2002) y para SGO-PV 1063 según Acosta-Hospitaleche \& Canto (2005). Medidas expresadas en mm

Comparison of measures and cranial indexes of Spheniscus and one specimen conferred to Palaeospheniscus. Measures for the genus Spheniscus sensu Stucchi (2002) and for SGO-PV 1063 sesu Acosta-Hospitaleche \& Canto (2005). Measures in millimeters

\begin{tabular}{|c|c|c|c|c|c|}
\hline Medidas & \multirow{2}{*}{$\begin{array}{c}\text { cf. Palaeospheniscus } \\
\text { SGO-PV } 1063 \\
47,2\end{array}$} & \multirow{2}{*}{$\begin{array}{c}\text { S. urbinai } \\
\text { MUSM } 269 \\
45,3\end{array}$} & \multirow{2}{*}{$\begin{array}{c}\text { S. megaramphus } \\
\text { MUSM } 175 \\
40,7\end{array}$} & \multirow{2}{*}{$\begin{array}{c}\text { S. humboldti } \\
32,6\end{array}$} & \multirow{2}{*}{$\begin{array}{c}\text { S. magallanicus } \\
32,8\end{array}$} \\
\hline 1 Altura & & & & & \\
\hline 2 Ancho postorbital & 55,8 & 73,5 & 73,9 & 49,7 & 50,7 \\
\hline 3 Ancho región occipital & 55 & 58 & 58,7 & 39,5 & 40,4 \\
\hline
\end{tabular}

Índice

\begin{tabular}{lllllll} 
A & $2 / 1$ & 1,1 & 1,6 & 1,8 & 1,5 & 1,5 \\
B & $2 / 3$ & 0,9 & 1,2 & 1,2 & 1,2 & 1,2 \\
C & $3 / 1$ & 1,1 & 1,2 & 1,4 & 1,2 & 1,2 \\
\hline
\end{tabular}


índices ligeramente menores a los obtenidos en el género Spheniscus. Es importante señalar que las medidas 1 y 3 parecen estar subestimadas, dado que en la ilustración se aprecia que ni la región occipital ni la postorbital se encuentran bien conservadas, lo que podría originar las diferencias obtenidas en los índices aplicados. De momento y en base a la información publicada, resulta imposible realizar análisis estadísticos para hallar diferencias significativas, sin embargo se espera que futuros estudios morfométricos permitan una mejor evaluación numérica.

Con base en lo anteriormente expuesto y teniendo en cuenta que el género Palaeospheniscus fue tipificado a partir de elementos postcraneales, los cuales no han sido hallados directamente asociados a materiales craneales (Acosta-Hospitaleche 2004), se descarta la identificación original presentada por Acosta-Hospitaleche \& Canto (2005) y se sugiere la inclusión de los especímenes en el género Spheniscus. La ausencia de elementos rostrales asociados, impide realizar una diferenciación específica de los especímenes.

De este modo se reduce el número de registros en la formación de nueve a siete: (1) Spheniscus urbinai, (2) S. megaramphus, (3) S. spp, (4) Pygoscelis calderensis (AcostaHospitaleche et al. 2006), (5) P. grandis (Walsh \& Suárez 2006), (6) Sphenicidae indet. aff. Paraptenodytes y (7) Sphenicidae gen. indet. nov. sp. Se emplea la designación de $S$. spp. para los especímenes atribuidos previamente como $S$. cf. chilensis ${ }^{2}$ y $S$. aff. humboldti (Chavez 2005) ${ }^{6}$, a la espera de descripciones detalladas de ambos registros.

\section{AGRADECIMIENTOS}

Al Instituto de Zoología de la Universidad Austral de Chile y a la Sociedad Paleontológica de Chile por su colaboración en la publicación del presente comentario.

\section{LITERATURA CITADA}

ACOSTA-HOSPITALECHE C (2004) Los pingüinos (Aves, Sphenisciformes) fósiles de Patagonia. Sistemática, biogeografía y evolución. Tesis doctoral, Universidad Nacional de La Plata, La Plata, Argentina. $284 \mathrm{pp}$

ACOSTA-HOSPITALECHE C \& J CANTO (2005) Primer registro de cráneos asignados a Palaeospheniscus (Aves, Spheniscidae) procedentes de la Formación Bahía Inglesa (Mioceno medio-tardío), Chile. Revista Chilena de Historia Natural 78: 489-495.

ACOSTA-HOSPITALECHE C, M CHÁVEZ \& O FRITIS (2006) Pingüinos fósiles (Pygoscelis calderensis nov. sp.) en la Formación Bahía Inglesa (Mioceno Medio- Plioceno), Chile. Revista Geológica de Chile 33: 327-338.

FRITIS O (2001) Descripción y análisis de la ornitofauna fósil de la formación Bahía Inglesa (Mioceno superior), III Región de Atacama, Chile. Tesis de grado, Departamento Ciencias de la Tierra, Universidad de Concepción, Concepción, Chile. 43 $\mathrm{pp}$

STUCCHI M (2002) Una nueva especie de Spheniscus (Aves: Spheniscidae) de la formación Pisco, Perú. Boletín de la Sociedad Geológica del Perú 94: 1724

STUCCHI M, M URBINA \& A GIRALDO (2003) Una nueva especie de Spheniscidae del Mioceno tardío de la Formación Pisco, Perú. Boletín de la Sociedad Geológica del Perú 94: 17-24.

WALSH S \& J HUME (2001) A new neogene marine avian assemblage from north-central Chile. Journal of Vertebrate Paleontology 21: 484-491.

WALSH S \& M SUAREZ (2006) New penguin remains from the Pliocene of northern Chile. Historical Biology 18: 115-126.

6 CHÁVEZ M (2005) Nuevos registros de aves fósiles en la Formación Bahía Inglesa (Mioceno-plioceno), región de Atacama, Chile. Octavo Congreso Chileno de Ornitología, Chillán, Chile. Actas: 47 
